\title{
The Bolyai-Lobatschewsky Non-Euclidean Geometry : an Elementary Interpretation of this Geometry, and some Results which follow from this Interpretation.
}

By Professor H. S. Carslaw, Sc.D.

(Received December 1909. Read 11th March 1910.)

\section{\$1. Introductory.}

Much has been written in recent years on the foundations of geometry, chiefly in Germany and Italy, and the relations of the various Non-Euclidean geometries to the Euclidean system are now more generally known among mathematicians. But most of these writings involve a knowledge of more advanced mathematics, while it has been found difficult to represent even the simplest NonEuclidean geometry-that of Bolyai-Lobatschewsky-in an elementary manner.

As is well known, the discovery of this system was due to the long continued, but necessarily unsuccessful, attempts to prove the Euclidean hypothesis (Axiom 12) on which Euclid's Theory of Parallels rests. (Throughout this paper this hypothesis will usually be referred to as Euclid's Parallel Postulate, because its original place was almost certainly among the Postulates, and it. ranks with them as one of the assumptions of the system.) Towards the beginning of last century, Gauss, Lobatschewsky and Bolyai showed that all such efforts must be unsuccessful, for they established the logical possibility of a geometry, ${ }^{*}$ the foundations of

* For information upon the History of the Theory of Parallels, see specially -

Engel und Stäckel-Theorie der Parallellinien von Euklid bis Gauss (Leipzig, 1895) ; and Bonola - La Geometria Non-Euclidea-Esposizione storicocritica del suo sviluppo (Bologna, 1906). Also Gauss, Werke Bd. VIII.

Historical references will also be found in-

Withers-Euclid's Parallel Postulate (Chicago, 1905) ; Russell-An Es8ay on the Foundations of Geometry (Cambridge, 1897); Klein-Nichteuklidische Geometrie (Göttingen, 1893), and elsewhere. 
which are exactly the same as those of the Euclidean geometry, except that instead of the hypothesis that only one parallel can be drawn to a straight line through a point outside it-a hypothesis equivalent to Euclid's Parallel Postulate-they assumed that two such parallels could be drawn. If this Non-Euclidean geometry forms a logical system and does not lead to any contradictions, then the axioms upon which it is built are not contradictory. If this be the case, it would be impossible to prove Euclid's Parallel Postulate from the other assumptions made by him, since this would involve the denial of the Parallel Postulate of Bolyai and Lobatschewsky.

Various demonstrations have been given of the logical possibility of this and the other Non-Euclidean geometries. Some are analytical, and depend upon the fact that the analytical expression of these geometries gives rise to a set of consistent equations. Others are geometrical, involving a knowledge of projective geometry, or the geometry of the surfaces of constant curvature.*

It is the object of this paper to give a simple proof of this result, to understand which a knowledge only of elementary geometry will be required. This proof is based upon the properties of the system of spheres all orthogonal to a fixed sphere. It will be found that the Non-Euclidean geometry can be interpreted in the geometry of this system of spheres. Some of its well-known theorems will be obtained from the elementary properties of the system, and it will be shown that if any contradiction were to arise in the NonEuclidean geometry, such a contradiction would also necessarily appear in the geometry of the system of spheres, and therefore in the Euclidean geometry. $\dagger$

* C. Bonola, loc. cit., $\$ 94$.

$\uparrow$ The idea of representing the Non-Fuclidean plane on the Euclidean half-plane, so that semicircles cutting the axis of $x$ at right angles take the place of straight lines, is due primarily to Klein and Poincaré. An analytical treatment on these lines will be found in Liebmann's Nichteuklidische Qeometrie (Leipzig, 1905). The system of spheres orthogonal to a fixed sphere, which includes the system of spheres orthogonal to a plane as a special case, is adopted by Wellstein in Weber.Wellstein's Encyclopädie der ElementarMathematik, Vol. II., \$§8-11 (Leipzig, 1905). This paper was suggested by Wellstein's discussion, of which it may be regarded as an extension. 


\section{\$2. The system of circles passing through a fixed point.}

We shall examine first of all the representation of ordinary Euclidean geometry contained in the geometry of the system of spheres all passing through a fixed point. In plane geometry this reduces to the system of circles through a fixed point, and we shall begin with this case.

Since the system of circles through a point $O$ is the inverse of the system of straight lines lying in a plane, to every circle there corresponds a straight line, and the circles intersect at the same angle as the corresponding lines. The properties of the set of circles could be established from the knowledge of the geometry of the straight lines, and every proposition concerning points and straight lines in the one geometry could at once be interpreted as a proposition concerning points and circles in the other.

There is another way in which the geometry of these circles can be established independently. We proceed to describe this method, and we shall see later that from this representation of the Euclidean geometry, we shall be able to pass easily to a corresponding representation of the Non-Euclidean Geometry.

\section{\$3. Ideal Lines.}

It will be convenient to speak of the plane of the straight lines and the plane of the circles as two separate planes. We have seen that to every straight line in the plane of the straight lines there corresponds a circle in the plane of the circles. We shall call these circles ideal lines. The ideal points will be the same as ordinary points, except that the point $O$ will be excluded from the domain of the ideal points.

On this understanding we can say that any two different ideal points $A, B$ determine the ideal line $A B$, just as any two different points $A, B$ determine the straight line $A B$.

As the angle between the circles in the one plane is equal to the angle between the corresponding straight lines in the other, we define the angle between two ideal lines as the angle between the corresponding straight lines. Thus we can speak of ideal lines being perpendicular to each other or cutting at any angle.

\section{\$4. Ideal Parallel Lines.}

Let BC (Fig. 1) be any straight line, and A a point not lying upon it. 
Let $\mathrm{AM}$ be the perpendicular to $\mathrm{BC}$, and $\mathrm{AM}_{1}, \mathbf{A M}_{2} \ldots$ different positions of the line $\mathrm{AM}$ as it revolves from the perpendicular position through two right angles.

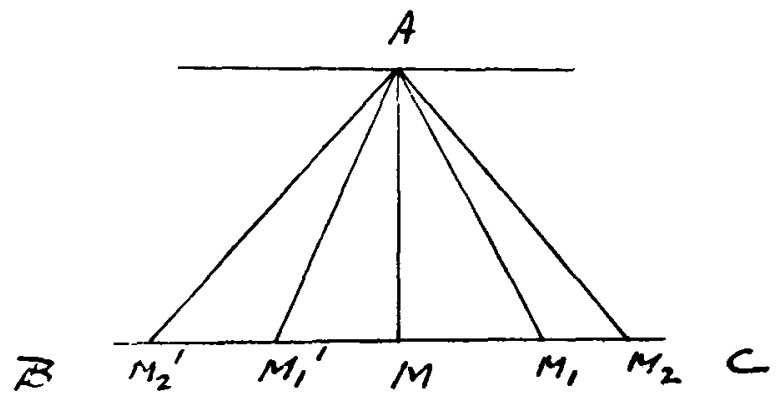

Fig. 1.

The lines begin by cutting $\mathrm{BC}$ on the one side of $\mathrm{M}$, and there is one line separating these lines which intersect $\mathrm{BC}$ on the one side from those which intersect it on the other. This line is the parallel through $\mathrm{A}$ to $\mathrm{BC}$.

In the corresponding figure for the ideal lines (Fig. 2) we have the ideal line through $A$ perpendicular to the ideal line $B C$ : and the circle, which passes through $A$ and touches the circle $O B C$ at $O$, separates the circles through $A$ which cut $B C$ on the one side of $M$ from those which cut it on the other.

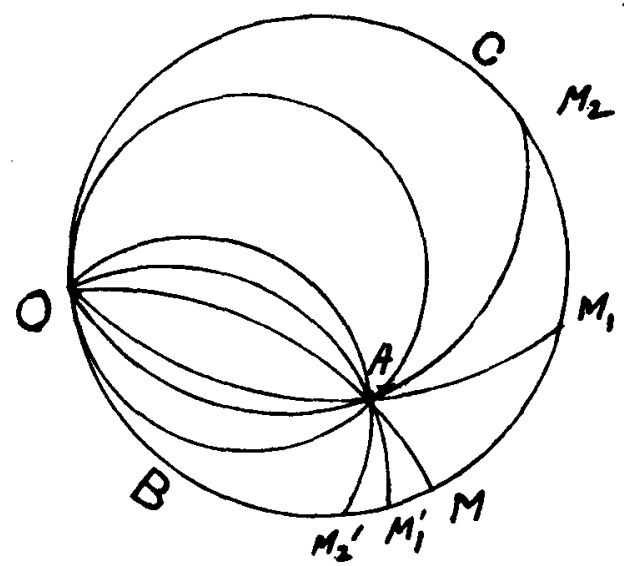

Fig. 2. 
We are thus led to define parallel ideal lines as follows:-

The ideal line through any point parallel to a given ideal line is the circle of the system which touches at $O$ the circle coinciding with the given ideal line, and passes through the given point.

Thus any two circles of the system which touch each other at $O$ will be ideal parallel lines. Two ideal lines, which are each parallel to a third ideal line, are parallel to each other, etc.

\section{§5. Ideal Lengths.}

Since Euclid's Parallel Postulate is equivalent to the assumption that one, and only one, straight line can be drawn through a point parallel to another straight line, and since this postulate is obviously satisfied by the ideal lines, Euclid's Theory of Parallels will be true in the geometry of these lines.

But such a geometry will require a measurement of length. We must now define what is meant by the ideal length of an ideal segment. In other words, we must define the ideal distance between two points. It is clear that, if the two geometries are to be identical, two ideal segments must be regarded as of equal length when the corresponding rectilinear segments are equal. We define the ideal lengths of an ideal segment as the length of the rectilinear segment to which it corresponds.

It will be seen that the ideal distance between two points $A, B$ is such that if $C$ is any other point on the segment

$$
\text { "distance" } \mathrm{AB}=\text { "distance" } \mathrm{AC}+\text { "distance" } \mathrm{CB} \text {. }
$$

The other requisite for distance is that it should be unaltered by displacement, and when we come to define Ideal Displacement we shall have to make sure that this condition is also satisfied.

It is clear that on this understanding the ideal length of an ideal line is infinite. If we take "equal" steps along the ideal line $\mathrm{BC}$ from the foot of the perpendicular (Fig. 2), the actual lengths of the arcs $\mathbf{M M}_{1}, M_{1} \mathbf{M}_{2}, \ldots$, if their ideal lengths are equal, become gradually smaller and smaller, as we proceed along the line towards $O$. It will take an infinite number of such steps to reach $O$, just as it will take an infinite number of steps along $B C$ from $\mathrm{M}$ (Fig. 1) to reach the point at which $\mathrm{BC}$ is met by the parallel through $A$. We have already seen that the domain of ideal points contains all the points of the plane except $O$. This was required so that the ideal line might always be determined by two different 
points. It is also needed for the idea of "between-ness." On the straight line $A B$ we can say that $C$ lies between $A$ and $B$ if we pass through $C$ as we proceed along $A B$ from $A$ to $B$. On the ideal line $\mathrm{AB}$ (Fig. 3) the points $\mathrm{C}_{1}$ and $\mathrm{C}_{2}$ would both lie between $\mathrm{A}$ and

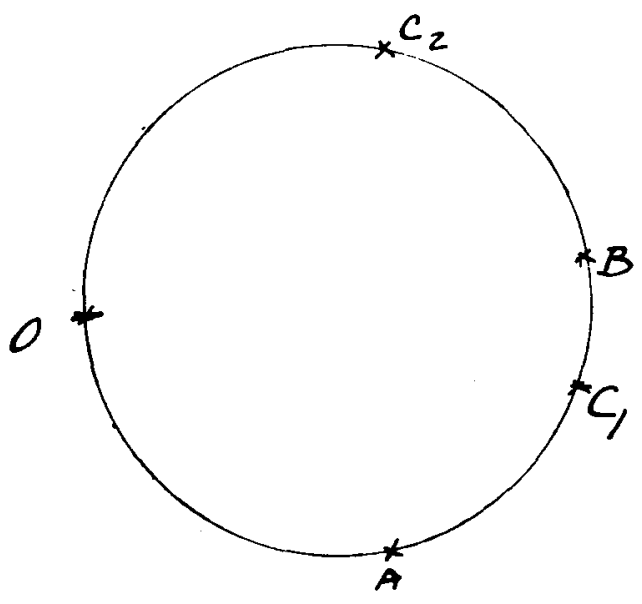

Fig. 3.

$B$, unless the point $O$ were excluded. In other words, this convention must be made so that the axioms of order* may appear in the geometry of the ideal points and lines.

On this understanding, and speaking still only of plane geometry, we can say that two ideal lines are parallel when they do not meet, however far they are produced.

To obtain an expression for the ideal length of an ideal segment, we may take the radius of inversion $k$ as unity.

Consider the ideal segment $\mathbf{A B}$ and the rectilinear segment $\alpha \beta$ to which it corresponds.

* Cf. Hilbert, Grundlagen der Geometrie, \$3 ; (Leipzig, 1899). English Translation, The Foundations of Geometry, p. 5 ; (Chicago, 1902). 
Then we have (Fig. 4) from similar triangles,

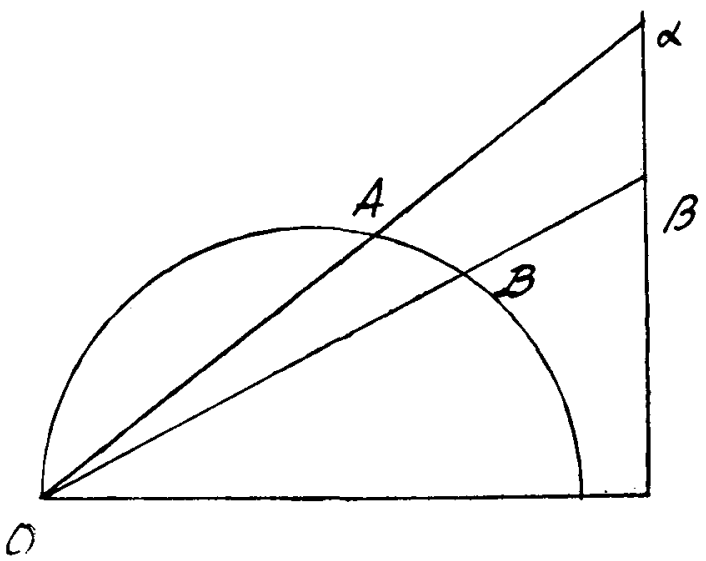

Fig. 4.

$$
\begin{aligned}
\frac{\alpha \beta}{\mathrm{AB}} & =\frac{\mathrm{O} \beta}{\mathrm{OA}} \\
& =\frac{\mathrm{O} \beta \cdot \mathrm{OB}}{\mathrm{OA} \cdot \mathrm{OB}} \\
& =\frac{k^{2}}{\mathrm{OA} \cdot \mathrm{OB}} .
\end{aligned}
$$

Thus we may say that the ideal length of the segment $\mathrm{AB}$ is equal to $\frac{\mathrm{AB}}{\mathrm{OA} \cdot \mathrm{OB}}$.

We shall now show that the ideal length of an ideal segment is unaltered by inversion with regard to any circle of the system.

Let $O D$ be any circle of the system, and let $\mathrm{C}$ be its centre (Fig. 5).

Then inversion with regard to this circle changes an ideal line into an ideal line.

Let the ideal segment $\mathrm{AB}$ invert into the ideal segment $\mathrm{A}^{\prime} \mathrm{B}^{\prime}$. These two ideal lines intersect at the point $D$, where the circle of inversion meets $A B$ 


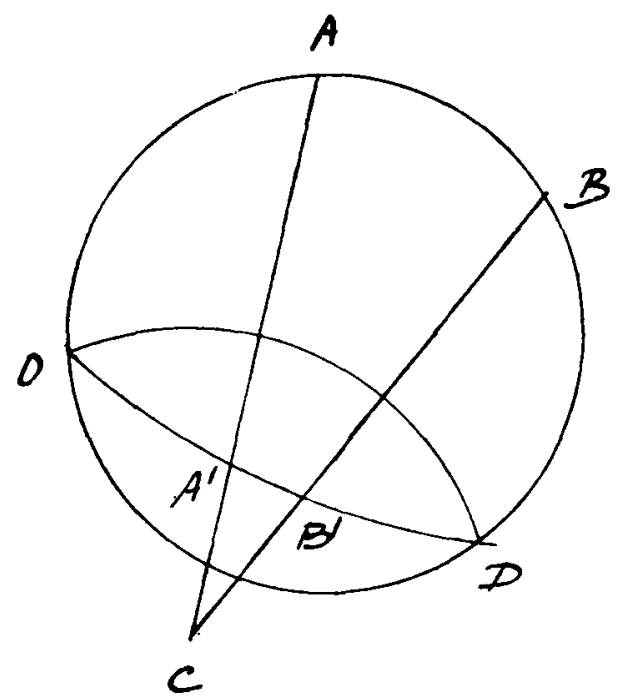

Fig. 5.

Then

$$
\begin{aligned}
& \frac{\text { the ideal length of } A D}{\text { the ideal length of } A^{\prime} D}=\frac{A D}{O A . O D} / \frac{A^{\prime} D}{O A^{\prime} \cdot O D} \\
& =\frac{\mathrm{AD}}{\mathbf{A}^{\prime} \mathbf{D}} \cdot \frac{\mathrm{OA}^{\prime}}{\mathrm{OA}}
\end{aligned}
$$

But from the triangles $C A D, C^{\prime} D$ and $O A C, O A^{\prime} C$ we find

$$
\frac{\mathrm{AD}}{\mathrm{A}^{\prime} \mathrm{D}}=\frac{\mathrm{CA}}{\mathrm{CD}}=\frac{\mathrm{CA}}{\mathrm{CO}}=\frac{\mathrm{OA}}{\mathrm{OA}^{\prime}} \text {. }
$$

Thus the ideal length of $A D$ is equal to the ideal length of $A^{\prime} D$. Similarly we find $B D$ and $B^{\prime} D$ have the same ideal length, and therefore $A B$ and $A^{\prime} B^{\prime}$ have the same ideal length.

\section{\$6. Ideal Displacements.}

The length of a segment must be unaltered by displacement. This leads us to consider the definition of ideal displacement. In his geometry Euclid uses the principle of displacement (e.g. let the triangle $A B C$ be superposed upon the triangle $A^{\prime} B^{\prime} C^{\prime}$, so that $A$ may coincide with $A^{\prime}$, etc.). In constructing this analogous geometry we shall use a corresponding principle. Any displacement 
in Euclidean geometry may be produced by repeated applications of reflection: that is, by taking the image of the figure in a line (or in a plane in the case of solid geometry).

For example, to translate the segment AB (Fig. 6) into another

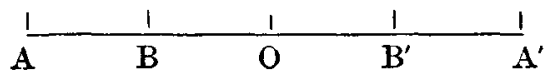

Fig. 6.

position on the same line, we may reflect the figure first about a line perpendicular to and bisecting $\mathrm{BB}^{\prime}$, and then another reflection about the middle point of $\mathrm{B}^{\prime} \mathrm{A}^{\prime}$ would bring the corresponding ends into their former positions relative to each other.

Also to move the segment $\mathrm{AB}$ into the position $\mathrm{A}^{\prime} \mathrm{B}^{\prime}$ (Fig. 7) we

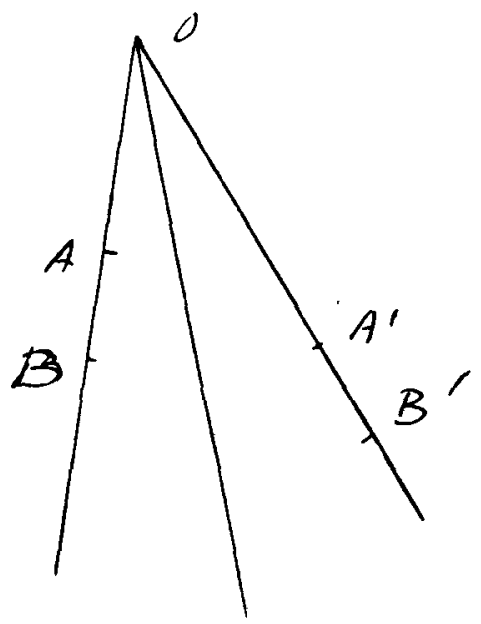

Fig. 7.

can first take the image of $\mathrm{AB}$ in the line bisecting the angle between $A B$ and $A^{\prime} B^{\prime}$, and then translate the segment along $A^{\prime} B^{\prime}$ to its final position.

We proceed to show that inversion with respect to any circle of the system is equivalent to reflection of the ideal points and lines in the ideal line which coincides urith the circle of inversion. 
Let $\mathrm{C}$ (Fig. 8 ) be the centre of any circle of the system, and let $A^{\prime}$ be the inverse of the point $A$ with regard to this circle. Then the circle $\mathrm{OAA}^{\prime}$ is orthogonal to the circle of inversion. In other words, such inversion changes any point $A$ into a point $A^{\prime}$ on the

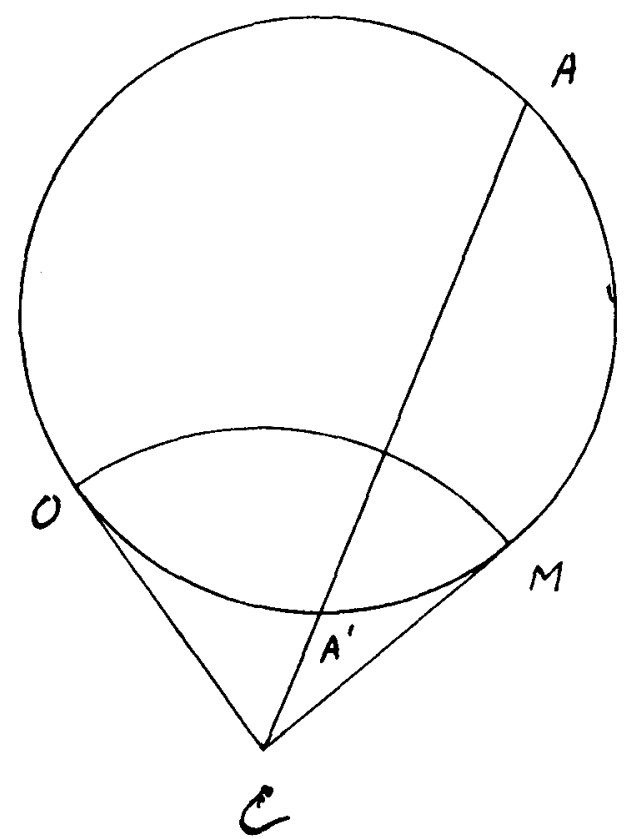

Fig. 8.

ideal line through A perpendicular to the circle of inversion. Also, the ideal line $\mathbf{A A}^{\prime}$ ' is " bisected" by that circle at $\mathbf{M}$, since the ideal segment $\mathbf{A M}$ inverts into the segment $\mathbf{A}^{\prime} \mathbf{M}$, and ideal lengths are unaltered by such inversion.

Again let $A B$ be any ideal segment, and by inversion with regard to any circle of this system let it take up the position $\mathbf{A}^{\prime} \mathbf{B}^{\prime}$ (Fig. 5).

We have seen that the ideal length of the segment is unaltered, and it is clear that the two segments when produced meet on the circle of inversion, and make equal angles with it. Also, the ideal lines $\mathrm{AA}^{\prime}$ and $\mathrm{BB}^{\prime}$ are perpendicular to, and bisected by, the ideal line with which the circle of inversion coincides. 
Such an inversion is therefore the same as reflection, and translation will occur as a special case of the above, when the circle of inversion is orthogonal to the given ideal line.

We thus define ideal reflection in an ideal line as inversion with this line as the circle of inversion.

It is unnecessary to say more about ideal displacements than that they will be the result of ideal reflections.

With these definitions it is now possible to "translate" every proposition in the ordinary plane geometry into a corresponding proposition in this ideal geometry. We have only to use the words ideal points, lines, parallels, etc., instead of the ordinary points, lines, parallels, etc. The argument employed in proving a theorem, or the construction used in solving a problem, will be applicable word for word in the one geometry as well as the other, since the elements involved satisfy the same laws. This may be called the "Dictionary Method," and is frequently met with in any discussion on Non-Euclidean geometry.*

\section{\$7. Extension to Solid Geometry. The system of spheres passing} through a fixed point.

These methods may be extended to solid geometry. In this case the inversion of the system of points, lines, and planes with regard to a sphere gives rise to the system of points, circles intersecting in the centre of inversion, and spheres also intersecting there. The geometry of this system of spheres could be derived from that of the system of points, lines, and planes by interpreting each proposition in terms of the inverse figures. For our purpose it is better to regard it as derived from the former by the invention of the terms ideal point, ideal lines, ideal planes, ideal lengths, and ideal displacements.

The ideal point is the same as the ordinary point, but the point $O$ is excluded from the domain of ideal points.

The ideal line through two ideal points is the circle of the system which passes through these two points.

The ideal plane through three ideal points, not on an ideal line, is the sphere of the system through these three points.

"Cf. Bonola, loc. oit., pp. 134, 155, 159, oto.

Poincaré, La Science et l' Hypothese, p. 56. 
Thus the plane geometry discussed in the preceding articles is a special case of this plane geometry.

Ideal parallel lines are defined as before. The line through $\mathbf{A}$ parallel to $B C$ is the circle of the system, lying on the sphere through $\mathrm{O}, \mathrm{A}, \mathrm{B}$, and $\mathrm{C}$, which touches the circle given by the ideal line $\mathrm{BC}$ at $\mathrm{O}$ and passes through $\mathrm{A}$.

It is clear that an ideal line is determined by two points, as a straight line is determined by two points. The ideal plane is determined by three points, not on an ideal line, as an ordinary plane is determined by three points, not on a straight line. If two points of an ideal line lie on an ideal plane, all the points of the line do so: just as if two points of a straight line lie on a plane, all its points do so. The intersection of two ideal planes is an ideal line, just as the intersection of two ordinary planes is a straight line.

The measurement of angles in the two spaces is the same.

For the measurement of length we adopt the same definition of ideal length as in the case of two dimensions. The ideal length of an ideal segment is the length of the rectilinear segment to which it corresponds.

To these definitions it only remains to add that of ideal displacement. As in the two dimensional cases, this is reached by means of ideal reflection. It can easily be shown that inversion with regard to any sphere of the system is equivalent to reflection of the ideal points, lines, and planes in the ideal plane coinciding with that sphere.

This ideal geometry is therefore identical with the ordinary Euclidean geometry. Its elements satisfy the same laws: every proposition valid in the one is also valid in the other: and from the theorems of the Euclidean geometry, those of the ideal geometry can be inferred.

In the articles that follow we shall establish an ideal geometry whose elements satisfy the axioms upon which the Non-Euclidean geometry of Bolyai-Lobatschewsky is based; that is, the set of axioms is the same, except that a new parallel axiom takes the place of the Euclidean hypothesis. The points, lines, and planes of this geometry will be figures of the Euclidean geometry: and from the known properties of these figures, we could state what the corresponding theorems of this Non-Euclidean geometry would 
be. Also from some of these Euclidean constructions, certain of the Non-Euclidean constructions could be obtained. This process would be the converse of that referred to in dealing with the ideal geometry of the preceding article. The theorems of the ideal geometry have there been obtained from the corresponding theorems in Euclid. We could in the same way obtain the theorems of the proposed ideal geometry from those of the Non-Euclidean geometry. If the Non-Euclidean geometry were to lead to any inconsistency, this would also arise in the ideal geometry. However, every step in the latter can be stated in the Euclidean fashion, and the contradiction would thus be bound to occur also in the Euclidean geometry. Since the latter is known to be a logically accurate system, it follows that this Non-Euclidean geometry is also logically accurate.

The Geometry of the System of Circles orthogonal to a Fixed Circle.

\section{\$8. Ideal Points, Ideal Lines, and Ideal Parallels.}

In the ideal geometry discussed in the previous articles, the ideal point was the same as the ordinary point, and the ideal lines and planes had so far the characteristics of straight lines and planes that they were lines and surfaces respectively. Geometries can be constructed in which the ideal points, lines, and planes are quite removed from ordinary points, lines, and planes, so that the ideal points no longer have the characteristic of being indivisible, and the ideal lines no longer boast only length, etc. What is required in each geometry is that the entities concerned satisfy the axioms which form the foundations of geometry. If they satisfy the axioms of Euclidean geometry, the arguments which lead to the theorems of that geometry will give corresponding theorems in the ideal geometry: and if they satisfy the axioms of any of the Non-Euclidean geometries, the arguments, which lead to theorems in that Non-Euclidean geometry, will lead equally to theorems in the corresponding ideal geometry.

We proceed to discuss the geometry of the system of circles orthogonal to a fixed circle.

Let this fundamental circle be of radius $k$ and centre $O$.

Let $A^{\prime}, A^{\prime \prime}$ be any two inverse points, $A^{\prime}$ being inside the circle. 
Every such pair of points $A^{\prime}, A^{\prime \prime}$ is an ideal point $(A)$ of the ideal geometry, with which we shall now deal.

If two of these pairs of points are given-that is, two ideal points, A, B-(fig. 9.), these determine a circle which is orthogonal to the fundamental circle.

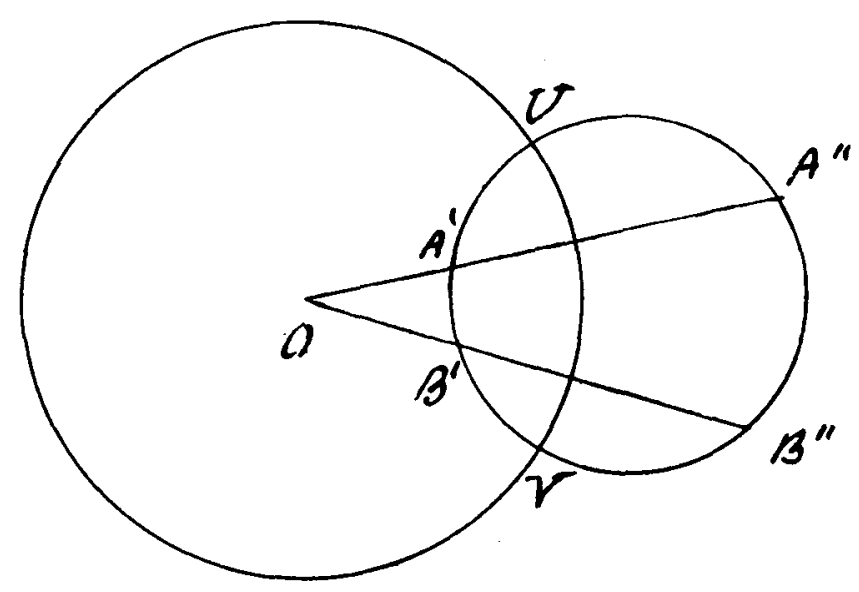

Fig. 9.

Every such circle is an ideal line of this ideal geometry.

Hence any two different ideal points determine an ideal line. In the case of the system of circles all passing through a fixed point $O$, this point $O$ was excluded from the domain of the ideal points. In this system of circles, all orthogonal to the fundamental circle, the coincident pairs of points lying on the circumference of that circle are excluded from the domain of the ideal points.

We define the angle between two ideal lines as the angle between the circles with which they coincide.

We have now to consider in what way it will be proper to define parallel ideal lines.

Let $A M$ be the ideal line through $A$ perpendicular to the ideal line $\mathrm{BC}$ : in other words, the circle of the system passing through $A^{\prime} A^{\prime \prime}$ and orthogonal to the circle through $B^{\prime} B^{\prime \prime} C^{\prime} C^{\prime \prime}$. (Fig. 10.) 


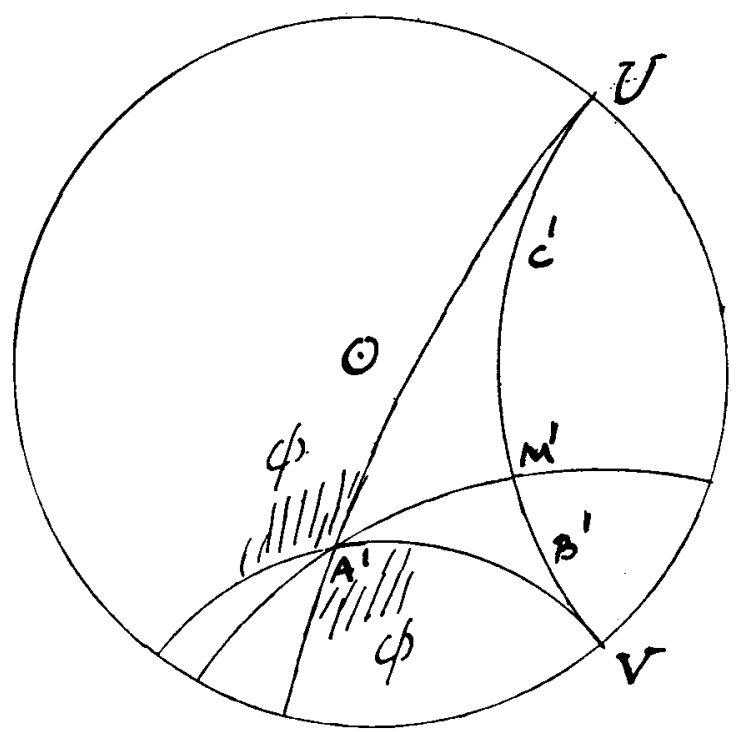

Fig. 10.

Imagine AM to rotate about $A$ so that these ideal lines through $A$ cut the ideal line $\mathrm{BC}$ at a gradually smaller angle. The circles through $A$ which touch the given circle $B C$ at the points $U, V$, where it meets the fundamental circle, are ideal lines of the system. They separate the lines of the pencil of ideal lines through $\mathbf{A}$ which cut the ideal line BC, from those which do not cut that line. All the lines in the angle $\phi$, shaded in the figure, do not cut the line BC : all these in the angle $\psi$, unshaded, cut this line.

This property is exactly what is assumed in the parallel postulate upon which the Non-Euclidean geometry of BolyaiLobatschewsky is based. We are therefore led to define the parallel ideal lines of this plane ideal geometry as follows :-

The ideal lines through an ideal point parallel to a given ideal line are the two circles of the system passing through the given point, which touch the circle with which the given line coincides at the points where it meets the fundamental circle.

Thus we have, in this ideal geometry, two parallels through a point to a given line-a right-handed parallel and a left-handed parallel, and these separate the lines of the pencil which intersect the given line from those which do not intersect it. 
\$9. Some Theorems of this Non-Euclidean Geometry.

At this stage we can say that any of the theorems of the Bolyai-Lobatschewsky Non-Euclidean geometry involving only angle properties will hold in this ideal geometry, and vice versa. Those involving lengths we cannot yet discuss, as we have not defined ideal lengths. For example, it is obvious that there are triangles in which all the angles are zero. (Fig. 11.) The sides of

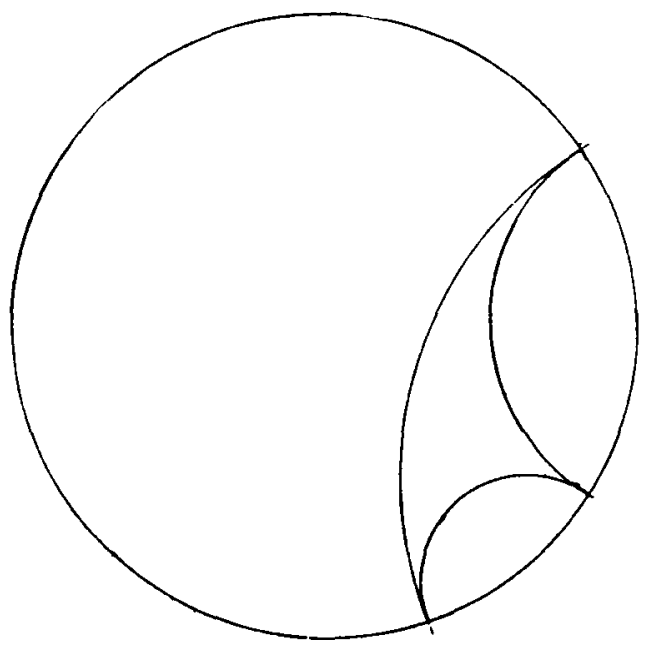

Fig. 11.

such triangles are parallel in pairs. Thus the sum of the angles of an ideal triangle is certainly not always two right angles. We can prove that this sum is always less than two right angles by a simple application of inversion, as follows:-

Let $\mathrm{C}_{1}, \mathrm{C}_{2}, \mathrm{C}_{3}$ be three circles of the system, forming an ideal triangle. Invert these circles from the point of intersection $I$ of the circles $\mathrm{C}_{1}$ and $\mathrm{C}_{2}$ which lies inside the fundamental circle. Then $\mathrm{C}_{1}$ and $\mathrm{C}_{2}$ become two straight lines $\mathrm{C}_{1}^{\prime}$ and $\mathrm{C}_{2}^{\prime}$ through $\mathrm{I}$. Also the fundamental circle $\mathrm{C}$ inverts into a circle $\mathrm{C}^{\prime}$ cutting $\mathrm{C}_{1}{ }^{\prime}$ and $\mathrm{C}_{2}^{\prime}$ at right angles, so that its centre is $\mathrm{I}$. Again, the circle $\mathrm{C}_{3}$ inverts into a circle $\mathrm{C}_{3}^{\prime}$, cutting $\mathrm{C}^{\prime}$ at right angles. Hence its centre lies outside $\mathrm{C}^{\prime}$. We thus obtain a "triangle" of which the 
sum of the angles is less than two right angles, and since these angles are equal to the angles of the ideal triangle, this result also holds for the ideal triangle.

Finally, it can easily be shown that there is always one, and only one, circle of the system cutting two non-intersecting circles of the system at right angles. In other words, two non-intersecting ideal lines have a common perpendicular,

All these results must be true in this Non-Euclidean geometry.

\$10. Ideal Lengths and Ideal Displacements.

Before we can proceed to the discussion of the metrical properties of this geometry we must define the ideal length of an ideal segment. It is clear that this must be such that it will be unaltered if we take the points $A^{\prime \prime}, B^{\prime \prime}$ as defining the segment $A, B$, instead of the points $A^{\prime}, B^{\prime}$. It must make the complete line of infinite length. It must satisfy the distributive law that "distance" $\mathrm{AB}=$ "distance" $\mathrm{AC}+$ "distance" $\mathrm{CB}$, if $\mathrm{C}$ is any other point on the segment $\mathrm{AB}$ : and it must also remain unaltered by ideal displacement.

We define the ideal lengths of any segment as follows :-

The ideal length of any segment $A B$ is equal to

$$
\log \left(\frac{\mathbf{A}^{\prime} \mathbf{V}}{\mathbf{A}^{\prime} \mathrm{U}} / \frac{\mathbf{B}^{\prime} \mathbf{V}}{\overline{\mathbf{B}^{\prime} \mathbf{U}}}\right)
$$

where $U$ and $V$ are the points where the ideal line $A B$ meets the fundamental circle. (Fig. 9.)

This expression obviously involves the an-harmonic ratio of the points $U, A, B, V$, and its connection with Cayley's definition of distance by means of the Absolute is evident.

It will be seen that this definition satisfies the first three of the conditions named above. It remains for us to examine what must represent displacement in the ideal geometry.

Let us consider what is the effect of inversion with regard to a circle of the system upon the system of ideal points and lines.

Let $\left(A^{\prime}, A^{\prime \prime}\right)$ be any ideal point $A$ (Fig. 12). Let the circle of inversion meet the fundamental circle in $\mathrm{C}$, and let $\mathrm{D}$ be its centre.

Let $A^{\prime}, A^{\prime \prime}$ invert into $B^{\prime}, B^{\prime \prime}$. 


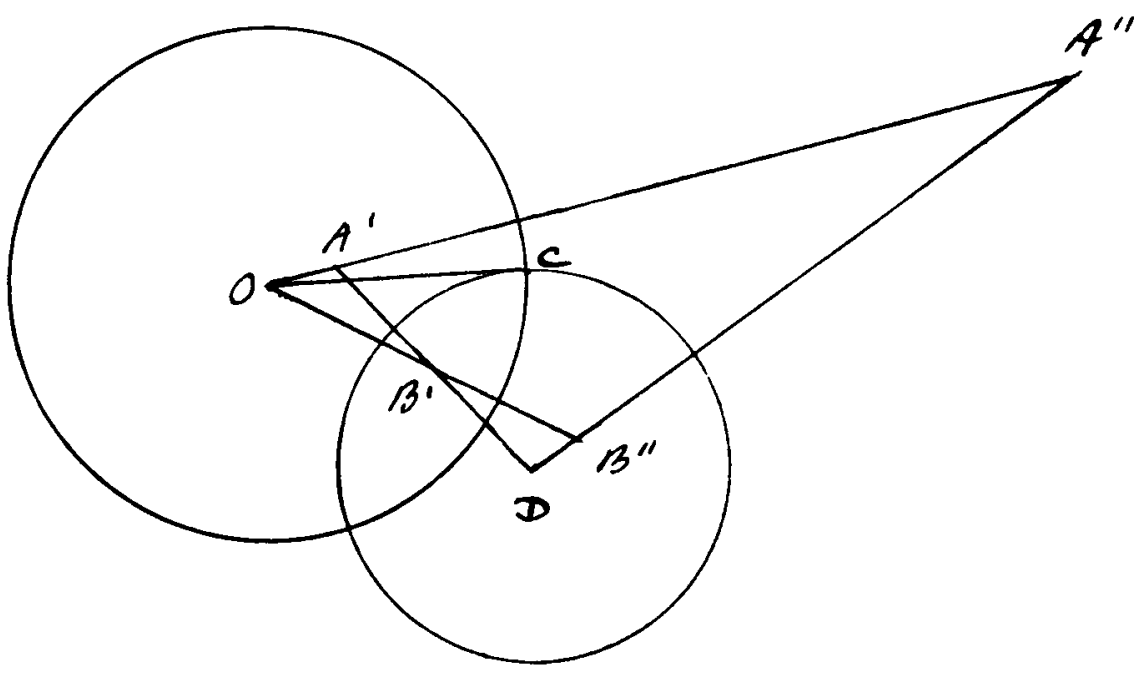

Fig. 12.

Since the circle $A^{\prime} A^{\prime \prime} C$ touches the circle of inversion at $C$, its inverse also touches that circle at $C$. But a circle passes through $A^{\prime}, A^{\prime \prime}, B^{\prime}, B^{\prime \prime}$, and the radical axes of the three circles

$$
A^{\prime} A^{\prime \prime} C, B^{\prime} B^{\prime \prime} C, A^{\prime} A^{\prime \prime} B^{\prime \prime} B^{\prime \prime}
$$

are concurrent.

Hence $B^{\prime} B^{\prime \prime}$ passes through $O$ and $\mathrm{OB}^{\prime} . \mathrm{OB}^{\prime \prime}=\mathrm{OC}^{2}$.

Therefore inversion with regard to any circle of the system changes an ideal point into an ideal point.

But it is clear that the circle $\mathbf{A}^{\prime} \mathbf{A}^{\prime \prime} \mathbf{B}^{\prime} \mathbf{B}^{\prime \prime}$ is orthogonal to the fundamental circle, and also to the circle of inversion.

Thus the ideal line joining the ideal point A to the ideal point B into which it is changed by this inversion is perpendicular to the ideal line coinciding with the circle of inversion.

We shall now prove that it is " bisected" by that ideal line.

Let the circle through $\mathbf{A B}$ meet the circle of inversion at $M$ and the fundamental circle in $U$ and $\nabla$. It is clear that $U, V$ are inverse points with regard to the circle of inversion (Fig. 13). 


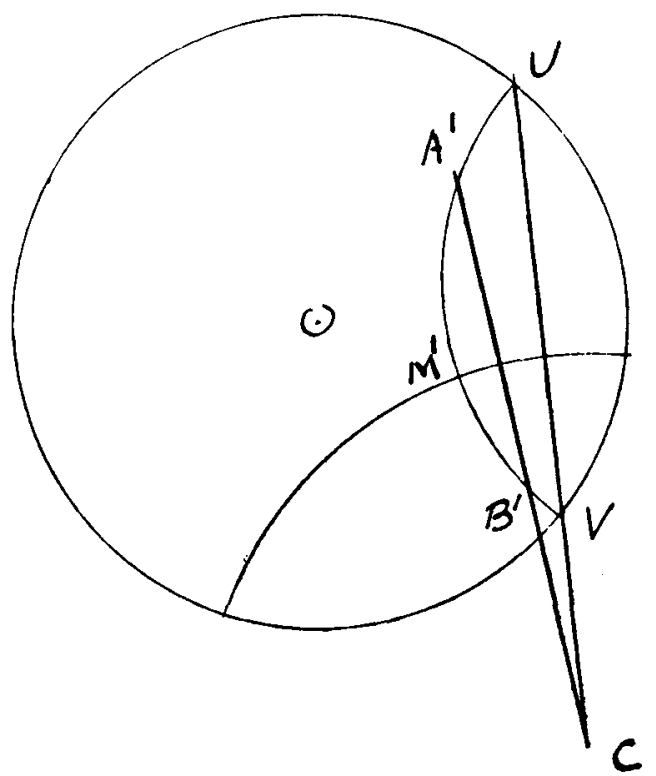

Then we have

Fig. 13.

$$
\begin{aligned}
& \frac{\mathbf{B}^{\prime} \mathbf{V}}{\mathbf{A}^{\prime} \mathrm{U}}=\frac{\mathrm{CV}}{\mathbf{C A}^{\prime}} \\
& \frac{\mathbf{A}^{\prime} \mathbf{V}}{\mathbf{B}^{\prime} \mathrm{U}}=\frac{\mathrm{CV}}{\mathbf{C} \mathbf{B}^{\prime}} \text {. } \\
& \therefore \quad \frac{\mathrm{A}^{\prime} \mathrm{V}}{\mathrm{A}^{\prime} \mathrm{U}} \cdot \frac{\mathrm{B}^{\prime} \mathrm{V}}{\mathrm{B}^{\prime} \mathrm{U}}=\frac{\mathrm{CV}^{2}}{\mathrm{CA}^{\prime} \cdot \mathrm{CB}^{\prime}}=\frac{\mathrm{CV}^{2}}{\mathrm{CM}^{\prime 2}}=\left(\frac{\mathrm{M}^{\prime} \mathrm{V}}{\bar{M}^{\prime} \mathrm{U}}\right)^{2} \text {. } \\
& \text { Therefore } \frac{\mathbf{A}^{\prime} \mathbf{V}}{\mathbf{A}^{\prime} \mathbf{U}} / \frac{\mathbf{M}^{\prime} \mathbf{V}}{\mathbf{M}^{\prime} \mathbf{U}}=\frac{\mathbf{M}^{\prime} \mathbf{V}}{\mathbf{M}^{\prime} \mathbf{U}} / \frac{\mathbf{B}^{\prime} \mathbf{V}}{\mathbf{B}^{\prime} \mathbf{U}} \text {. }
\end{aligned}
$$

Thus the ideal length of AM equal to the ideal length of BM.

Therefore we have the following result:-

Inversion with regard to any circle of the system changes any ideal point $A$ into an ideal point $B$ such that the ideal line $A B$ is perpendicular to, and "bisected" by, the ideal line coinciding with the circle of inversion.

In other words,

Inversion with regard to such a circle causes any ideal point to take the position of its image in the ideal line with which the circle of inversion coincides. 


\section{4}

We proceed to examine what effect such inversion has upon an ideal line.

Since a circle orthogonal to the fundamental circle inverts into a circle also orthogonal to the fundamental circle, any ideal line $\mathrm{AB}$ inverts into another ideal line $a b$ passing through the point $\mathbf{M}$ where $A B$ meets the circle of inversion (Fig. 14). Also the points

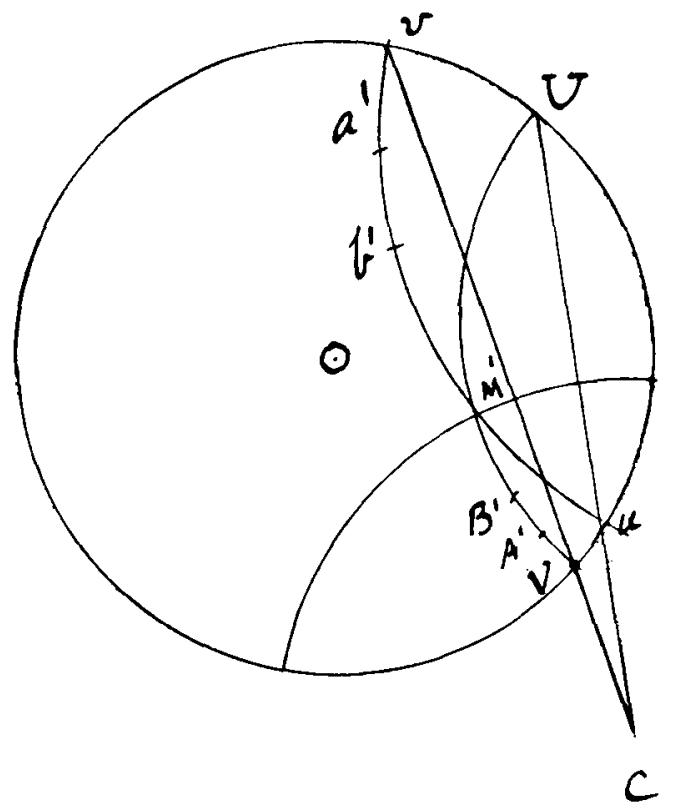

Fig. 14.

$\mathrm{U}, \mathrm{V}$ invert into the points $u, v$ on the fundamental circle, and the lines $\mathrm{AB}$ and $a b$ are equally inclined to the circle of inversion.

It is easy to show that the ideal lengths of $A M$ and $B M$ are equal to those of $a \mathrm{M}$ and $b \mathrm{M}$ respectively, and it follows that the ideal length of the segment $A B$ is unaltered by this inversion. Also, we have seen that $\mathrm{A} a$ and $\mathrm{B} b$ are perpendicular to, and bisected by, the ideal line coinciding with this circle.

We can thus state the following theorem :-

Inversion with regard to any circle of the system has the same effect upon an ideal segment as reflection in the corresponding ideal line. 
This discussion has therefore again led us to define ideal reflection in an ideal line as the inversion of this system of ideal points and lines with regard to the circle which coincides with this ideal line.

It is unnecessary to define ideal displacement as any displacement can be obtained by a series of reflections and any ideal displacement by a series of ideal reflections.

We notice that the definition of the ideal length of a segment fixes the ideal unit of length. We may take this unit segment on one of the diameters of the fundamental circle, since these lines are also ideal lines of the system. Let it be the segment OP (Fig. 15).

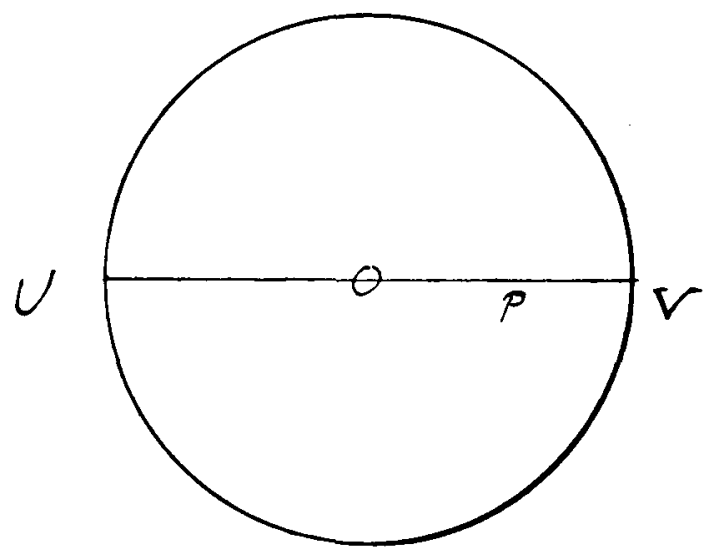

Fig. 15.

Then we must have

$$
\log \left(\frac{\mathrm{OV}}{\mathrm{OU}} / \frac{\mathrm{PV}}{\mathrm{PU}}\right)=1
$$

that is,

$$
\log \left(\frac{\mathrm{PU}}{\mathrm{PV}}\right)=1
$$

that is,

$$
\frac{\mathrm{PU}}{\mathrm{PV}}=e \text {. }
$$

Thus the point $\mathbf{P}$ divides the diameter in the ratio $e: 1$.

The unit segment is thus fixed for any position in the domain of the ideal points, since the segment $O P$ can be "moved" so that one of its ends coincides with any given ideal point. 
A different expression for the ideal length, viz.,

$$
k \log \left(\frac{\mathrm{AV}}{\mathrm{AU}} / \frac{\mathrm{BV}}{\mathrm{BU}}\right)
$$

would simply mean an alteration in this unit, and taking logarithms to the base $a$ instead of $e$ would have the same effect.

\section{\$11. Some further Theorems in the Hyperbolic Geonetry.}

We are now in a position to establish some further theorems in hyperbolic geometry, as the Bolyai-Lobatschewsky system is called.

In the first place we can state that similar triangles are impossible in this geometry. Indeed Saccheri had shown before its discovery* that, if the existence of only two equiangular triangles, which were not congruent, could be proved, then the Euclidean geometry was alone possible.

We also see that parallel lines are asymptotic; that is, they continually approach each other. This is clear from the figure for ideal parallels and the definition of ideal length (Fig. 10).

Further, it is also obvious that as the point $A$ moves away along the perpendicular MA to the line BC (Fig. 10), the angle of parallelism diminishes from $\frac{\pi}{2}$ to zero in the limit.

Again we can prove that the angle of parallelism, $\Pi(p)$, for the segment $p$, is given by

$$
e^{-p}=\tan \left(\frac{\Pi(p)}{2}\right)
$$

Consider an ideal line and the parallel to it through a point $A$.

Let AM (Fig. 16) be the perpendicular to the given line MU and $\mathrm{AU}$ the parallel.

* Cf. Engel und Stäckel, loc. cit., p. 84. Bonola, loc. cit., p. 26. 
Let the figure be inverted from the point $\mathbf{M}^{\prime \prime}$, the radius of inversion being the tangent from $\mathbf{M}^{\prime \prime}$ to the fundamental circle.

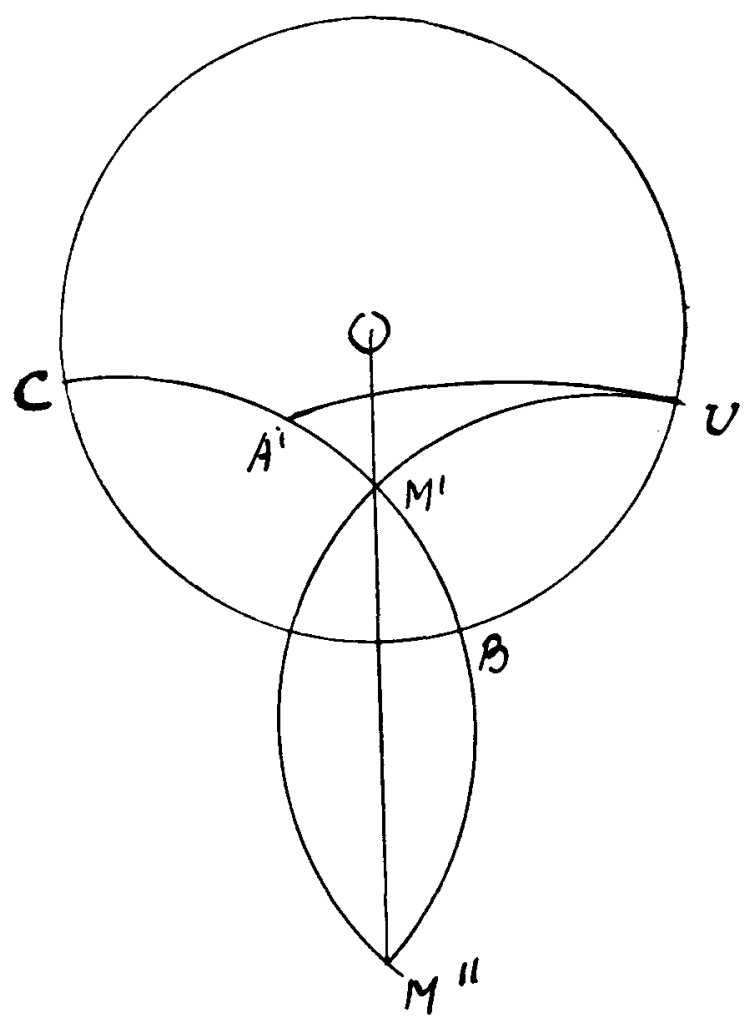

Fig. 16.

Then we obtain a new figure (Fig. 17) in which the corresponding ideal lengths are the same, since the circle of inversion is a circle of the system. The lines $\mathrm{AM}$ and $\mathrm{MU}$ become straight lines through the centre of the fundamental circle, which is the inverse of the point $\mathbf{M}^{\prime}$. Also, the circle $\mathrm{AU}$ becomes the circle $a^{\prime} u$, touching the radius $m^{\prime} u$ at $u$, and cutting $m^{\prime} a$ at an angle $\Pi(p)$. These radii $m^{\prime} u, m^{\prime} b$ are also ideal lines of the system.

Let the ideal length of $\mathrm{AM}$ be $p$. 


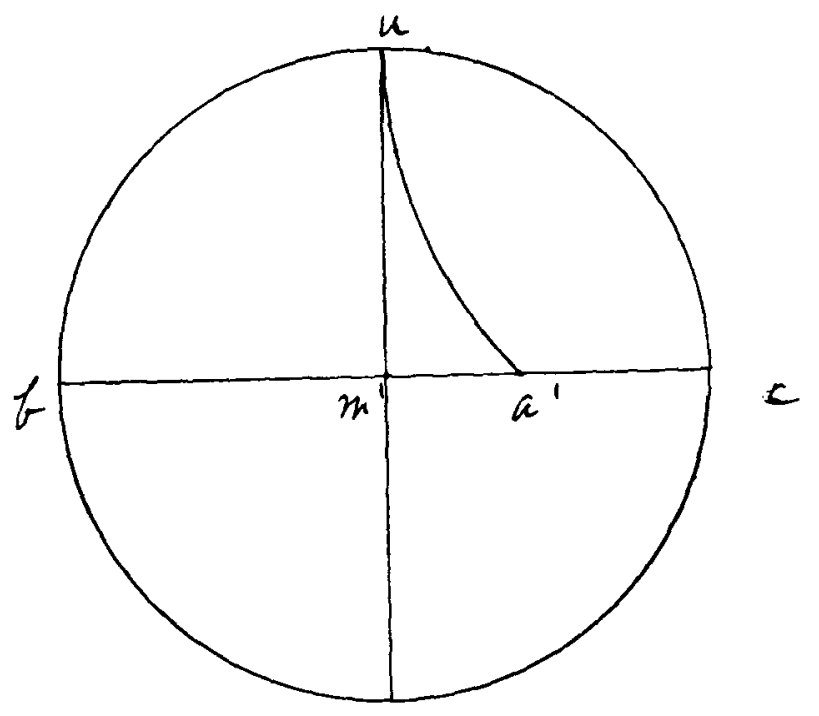

Fig. 17.

Then we have

$$
\begin{aligned}
p & =\log \left(\frac{\mathrm{A}^{\prime} \mathrm{B}}{\mathrm{A}^{\prime} \mathrm{C}} / \frac{\mathrm{M}^{\prime} \mathrm{B}}{\overline{\mathrm{M}^{\prime} \mathrm{C}}}\right) \\
& =\log \left(\frac{a^{\prime} b}{a^{\prime} \mathrm{c}} / \frac{m^{\prime} b}{m^{\prime} \mathrm{c}}\right) \\
& =\log \left(\frac{a^{\prime} b}{a^{\prime} c}\right) .
\end{aligned}
$$

But from the geometry of Fig. 17, remembering that $a^{\prime} u$ cuts $b c$ at the angle $\Pi(p)$, we have

$$
\begin{aligned}
& a^{\prime} c=k\left\{1-\tan \left(\frac{\pi}{4}-\frac{\Pi(p)}{2}\right)\right\}, \\
& a^{\prime} b=k\left\{1+\tan \left(\frac{\pi}{4}-\frac{\Pi(p)}{2}\right)\right\},
\end{aligned}
$$

where $k$ is the radius of the fundamental circle.

$$
\begin{aligned}
& \text { Therefure } \\
& p=\log \cot \left(\frac{\Pi(p)}{2}\right) \\
& \text { and } \\
& e^{-p}=\tan \left(\frac{\Pi(p)}{2}\right) \text {. }
\end{aligned}
$$


Finally in this geometry, there will be three kinds of circles. There will be (i) the circle with its centre at a finite distance; (ii) the limiting curve, with its centre at infinity, or at a point where two parallels meet; and (iii) the equidistant curve, with its centre at the imaginary point of intersection of two lines with a common perpendicular. The first of these curves would be traced out in the ideal geometry by one end of an ideal segment when it is reflected in the lines passing through its other end ; that is, by the rotation of this ideal segment about one of its ends. The second occurs when the ideal segment is reflected in the successive lines of the pencil of ideal lines all parallel to it in the same direction; and the third, when the reflection takes place in the system of lines which have all a common perpendicular with this segment. That these correspond to the circle, the limiting curve and the equidistant curve of the hyperbolic geometry, is easily proved.

\section{\$12. The Impossibility of proving Euclid's Parallel Postulate.}

We could obtain more of the results of the hyperbolic geometry, and find some of its constructions, by further examination of the properties of this set of circles, but this is not our object. Our argument was directed to proving that it is impossible for any contradiction to arise in this Non-Euclidean geometry. If such contradiction entered into this plane geometry, it would occur also in the interpretation of the result in the ideal geometry. Thus the contradiction would also be found in the Euclidean geometry. We can therefore state that it is impossible that any logical inconsistency could arise in the hyperbolic plane geometry. It could still be argued that such a contradiction might be found in the hyperbolic solid geometry. An answer to such an objection is forthcoming at once. The geometry of the system of circles, all orthogonal to a fixed circle, can be at once extended into a three dimensional system. The ideal points are taken as the pairs of points inverse to a fixed sphere, excluding the points on the surface of the sphere from their domain. The ideal lines are the circles through two ideal points. The ideal planes are the spheres through three ideal points, not lying on an ideal line. The ordinary plane enters as a particular case of these ideal planes, and so the plane geometry just 
discussed is a special case of a plane geometry of the system. With suitable definitions of ideal lengths, ideal parallels, and ideal displacements, we have a solid geometry exactly analogous to the hyperbolic solid geometry. It follows that no logical inconsistency could arise in the hyperbolic solid geometry, since, if such did occur, it would also be found in the interpretation of the result in this ideal geometry, and therefore it would enter into the Euclidean geometry.

By this result our argument is complete. However far the hyperbolic geometry were developed, no contradictory results could be obtained. This system is thus logically possible, and the axioms upon which it is founded are not contradictory. Hence it is impossible to prove Euclid's Parallel Postulate, since its proof would involve the denial of the Parallel Postulate of Bolyai and Lobatschewsky.

It should perhaps be pointed out that a more logical course to pursue would have been to show, with Hilbert, that Euclidean geometry is founded upon a certain set of axioms; and that the various Non-Euclidean geometries are derived by dropping one or other of these assumptions. It could then be shown that the first system of ideal points, lines, and planes discussed in \$1-7 satisfies the axioms on which the Euclidean geometry is based, and this establishes the correspondence between these two systems. It could also be shown that the second system of ideal points, lines, and planes used from $\S 8$ onwards, satisfies the same set of axioms, with the exception of the Euclidean Parallel Axiom for which the BolyaiLobatschewsky axiom is substituted. This ideal geometry is therefore equivalent to the hyperbolic geometry, and any inconsistency in the one would be found in the other.

However our object was to give a proof of the possibility of this Non-Euclidean geometry, and the consequent independence of Euclid's Parallel Axiom from the other axioms, that could be followed by any one who possessed only the ordinary knowledge of geometry obtained from an elementary mathematical course. For this reason no reference has been made to this part of Hilbert's work, and the principle of superposition has been introduced as in the ordinary elementary geometry. That this principle involves the axiom of free mobility, or a system of axioms of congruence, is true; but these matters are outside the plan of this discussion. 